\title{
Vivências, Habilidades Sociais e Comportamentos Sociais de Universitários
}

\author{
Adriana Benevides Soares ${ }^{1, ~}{ }^{\text {, }}$, Ana Maria Porto ${ }^{1}$, Claudio Almeida Lima ${ }^{1}$, Clystine Gomes ${ }^{1}$, \\ Denise Amorim Rodrigues ${ }^{1}$, Reivani Zanoteli ${ }^{2}$, Zeimara Almeida Santos ${ }^{1}$, \\ Alexandra Fernandes ${ }^{1}, \&$ Humberto Medeiros ${ }^{1}$ \\ ${ }^{1}$ Universidade Salgado de Oliveira, Rio de Janeiro, RJ, Brasil \\ ${ }^{2}$ Universidade Veiga de Almeida, Rio de Janeiro, RJ, Brasil
}

\begin{abstract}
RESUMO - Pode-se supor que a adaptação ao contexto universitário requeira tanto habilidades de relacionamento como comportamentos acadêmicos adequados. Este estudo destinou-se a identificar as relações entre Habilidades Sociais, Vivências Acadêmicas e Comportamentos Sociais Acadêmicos em estudantes. Participaram do estudo 289 estudantes universitários de instituições públicas e privadas, com idades variando entre 17 e 61 anos. Aplicou-se o Inventário de Habilidades Sociais (IHS), o Questionário de Vivências Acadêmicas (QVA-r) e o Inventário de Comportamentos Sociais Acadêmicos (ICSA). Foi identificado que os alunos que apresentam comportamentos sociais acadêmicos desadaptativos também apresentam déficits em habilidades sociais dificultando sua adaptação acadêmica e que, habilidades socialmente aceitáveis estão associadas não só a varáveis pessoais, mas também às curriculares e institucionais.
\end{abstract}

PALAVRAS-CHAVE: habilidades sociais, comportamentos sociais acadêmicos, universitários, ensino superior, adaptação acadêmica

\section{Livings, Social Skills and Social Behaviors of College Students}

\begin{abstract}
It can be assumed that adapting to the university context requires both relationship skills and appropriate academic behaviors. This study was designed to identify the relationship between Social Skills, Academic Experiences and Academic Social Behaviors in students. Study participants were 289 university students from public and private institutions, ranging between 17 and 61 years. We used the Social Skills Inventory (IHS), the Academic Experiences Questionnaire (QVA-r), and the Inventory of Academic Social Behaviors (ICSA). It was identified that students who have academic maladaptive social behaviors also have deficits in social skills hindering their academic adaptation and that, socially acceptable skills are linked to not only personal variables, but also to the curricular and institutional ones.
\end{abstract}

KEYWORDS: social skills, social behaviors academic, college, higher education, academic adaptation

Ao longo da última década, dado o aumento considerável de estudantes universitários, nota-se uma constante preocupação por parte das Instituições de Ensino Superior (IES) no que tange a sua estrutura física: espaços de conhecimento tais como laboratórios de informática bem equipados, bibliotecas atualizadas e qualificação do corpo docente (Kanan \& Zanelli, 2011). Estudos recentes têm sinalizado que, além das realidades estruturais, os recursos pessoais trazidos pelos alunos são determinantes no processo de aprendizagem e permanência na universidade e que a falta de disciplina, a má administração do tempo, o método de estudo inadequado e a inabilidade para as relações interpessoais são problemas que precisam ser considerados e combatidos permanentemente (Almeida, Soares, \& Ferreira, 2002). A maturidade psicossocial e a preparação acadêmica dos estudantes, segundo Costa e Oliveira (2010), influem de maneira significativa no processo de adaptação, pois capacidades pessoais como resiliência, objetivos claros, alcance de autonomia e domínio do conhecimento são facilitadores desse processo. Segundo Nunes (2005), os programas governamentais de inserção nas IES, o Fundo de Financiamento Estudantil (FIES), o ProUni, o Sistema

*E-mail: adribenevides@gmail.com 
de Cotas e a grande abertura de vagas nas universidades privadas de modo geral, desenvolvem-se na perspectiva de captar novos alunos sem se preocupar o suficiente com a sua retenção. A perda dos alunos ainda é tratada como uma decorrência natural, sendo aceitável que os alunos sem condições acadêmicas, financeiras ou psicológicas não concluam o seu curso (Nunes, 2005).

Nas pesquisas realizadas por T. W. Alves e Alves (2009) sobre a evasão, podem-se observar, em países como a África do Sul, taxas de $40 \%$ de abandono dos estudantes universitários no primeiro ano de graduação. Nos Estados Unidos, esse número se aproxima dos 50\% entre os estudantes de Ensino Superior. Nas universidades brasileiras, o tema vem sendo tratado sob a perspectiva da observação do número de vagas oferecidas para os cursos, de quantos ocupam essas vagas e do número daqueles que são diplomados ao fim do curso escolhido, enquanto em instituições internacionais, os estudos giram em torno das causas de tais fenômenos (Teixeira, Castro, \& Piccolo, 2007). A evasão escolar, enquanto fenômeno que ocorre nas universidades de forma cada vez mais presente, traz como possíveis motivações a necessidade de trabalhar a questão vocacional e a dificuldade de adaptar-se a um novo estilo de ensino-aprendizagem (Silva \& Fleith 2005). Santos, Noronha, Amaro e Villar (2005), Mercuri e Polydoro (2003) destacam que a evasão nas universidades envolve fatores externos ou objetivos que se correlacionam com a própria instituição escolhida, tais como questões financeiras, o deslocamento até a universidade e o local em que o estudante passa a residir, bem como com fatores de ordem interna ou subjetiva, que se correlacionam a quanto os alunos estão satisfeitos, ao modo como acontecem as relações interpessoais e à escolha profissional, entre outros. Sob os aspectos internos ou subjetivos, Parker, Sumnerfeldt, Hogan e Majestic (2004) apontam que as dificuldades interpessoais, o novo método de ensino-aprendizado, a falta de recursos pessoais e a ausência do ambiente familiar e dos amigos podem propiciar a interrupção do processo acadêmico e deflagrar estados psicopatológicos. Almeida e Soares (2003) consideram que as relações interpessoais e a preparação pedagógica dos professores se refletem na aprendizagem, no rendimento acadêmico e na interação dos estudantes com o curso e com a instituição. Constata-se, sob a perspectiva das relações interpessoais no ambiente universitário, que as dificuldades de interação pessoal podem resultar em fracasso nas atividades acadêmicas e acarretar, além de distúrbios psicológicos, a desistência do curso (Bandeira \& Quaglia, 2006).

Sob a perspectiva do modo como o aluno vivencia e se comporta no ambiente universitário, Soares, Poubel e
Mello (2009) destacam a importância do desenvolvimento de habilidades sociais na formação dos estudantes como facilitador do desempenho técnico e social do aluno. Pesquisas realizadas por Z. Del Prette e Del Prette (2004) apontam que os estudantes universitários com elevados déficits de habilidades sociais apresentam menor qualidade de relações interpessoais saudáveis, dificultando o funcionamento social e a capacidade de adaptar-se.

Habilidades sociais (HS) podem ser compreendidas como o conjunto de classes e subclasses comportamentais que o indivíduo apresenta para atender às diversas demandas das situações interpessoais (Z. Del Prette \& Del Prette, 2001). Em estudo com 90 universitários, foi possível coletar informações acerca das relações interpessoais vivenciadas na universidade. Bariani e Pavani (2008) detectaram categorias e subcategorias indicadoras de dificuldades comportamentais: o comportamento individual do aluno e do professor, bem como a relação inadequada entre os alunos e entre alunos e professores. As HS vivenciadas neses relacionamentos, quando adequadas, segundo os autores, proporcionam um profícuo desempenho acadêmico e maior índice de satisfação pessoal. As HS, como recursos presentes no indivíduo, são elementos importantes a serem considerados enquanto facilitadores de uma adaptação mais segura. Habilidades de comunicação, civilidade, assertividade, empatia e expressão de sentimentos positivos ajudam no desempenho e na construção da competência social (Feitosa, Matos, Z. Del Prette, \& Del Prette, 2009; Gresham, 2009).

Espera-se que o estudante universitário se desenvolva de modo adequado e satisfatório no âmbito acadêmico, obtendo boas notas, engajando-se nas atividades curriculares e extracurriculares e no convívio social (Miller, 2005). Por parte da IES, importa considerar, segundo Vendramini et al. (2004), que, para o bom desempenho da vida acadêmica, haja interesse pelo desempenho do aluno desde o seu ingresso até o término. Para os mesmos autores, o apoio dispensado na formação técnica dos estudantes precisa vir acompanhado de uma sólida formação pessoal. O mesmo aponta Tinto (2012) ao considerar que as IES devem promover a aprendizagem e o sucesso do estudante enquanto prioridade, estando atentas aos variados aspectos que envolvem a vida acadêmica, pois o desenvolvimento do aluno acontece de forma gradual com preocupações, necessidades e demandas diferenciadas. A partir dessas considerações, o presente estudo busca investigar as relações existentes entre as vivências acadêmicas, as HS e os comportamentos sociais acadêmicos de estudantes universitários de instituições públicas e privadas. 


\section{MÉTODO}

\section{Participantes}

Participaram da pesquisa 289 estudantes universitários de oito instituições públicas $(\mathrm{n}=83 ; 28,7 \%)$ e 20 privadas $(\mathrm{n}=206 ; 71,3 \%) ; 130$ eram do sexo feminino (45\%) e 159 do masculino (55\%), com idade variando entre 17 e 61 anos $(\mathrm{M}=26,73$ e D.P. $=8,57)$.

\section{Instrumentos}

Questionário de Vivências Acadêmicas na versão reduzida (QVA-r; Almeida et al., 2002). Validado para o Brasil por Villar e Santos (2001), é composto de 55 itens distribuídos nas dimensões Pessoal (alpha de Cronbach $=0,84)$, Interpessoal (alpha de Cronbach $=0,82)$, Carreira (alpha de Cronbach $=0,86$ ), Estudo (alpha de Cronbach $=0,78)$ e Institucional (alpha de Cronbach $=0,77$ ). Dezessete desses componentes $(4,6,9,11,13,17,20,25,26,27,34$, $40,41,46,47,50$ e 55$)$ eram invertidos.

Inventário de Habilidades Sociais ( IHS; Z. Del Prette \& Del Prette, 2001). É um instrumento de autorrelato composto de 38 itens. O respondente deve avaliar a frequência com que reage a cada situação. Sua resposta pode variar de nunca ou raramente até sempre ou quase sempre, em uma escala de cinco itens. Está estruturado em cinco fatores, que são Habilidades de Enfrentamento e Autoafirmação com Risco (alpha de Cronbach =0,96), Autoafirmação na Expressão de Sentimento Positivo (alpha de Cronbach =0,86), Conversação e Desenvoltura Social (alpha de Cronbach =0,81), Autoexposição a Desconhecido e Situações Novas (alpha de Cronbach $=0,75$ ) e Autocontrole da Agressividade (alpha de Cronbach $=0,74$ ). Os itens 2, 8 , $9,13,17,18,19,22,23,24,33,34,36$ e 37 tem sua escala de pontuação invertida.
Inventário de Comportamentos Sociais Acadêmicos (ICSA; Soares, Mourão, \& Mello, 2011). É composto de 34 itens e seis fatores: Habilidade em Sala de Aula (alpha de Cronbach $=0,73)$, Comportamento Indisciplinado em Sala de Aula (alpha de Cronbach =0,81), Cordialidade no Relacionamento Interpessoal (alpha de Cronbach $=0,77$ ), Desrespeito a Professores e Colegas (alpha de Cronbach $=0,59$ ), Autoexposição e Assertividade (alpha de Cronbach =0,66) e Habilidade em Eficácia Acadêmica (alpha de Cronbach $=0,60$ ).

\section{Procedimentos de Coletas de Dados}

Os dados foram coletados sendo inicialmente aplicado o IHS (Z. Del Prette \& Del Prette, 2001), em seguida o QVA-r (Almeida et al., 2002) e posteriormente o ICSA (Soares et al., 2011). Foram coletados em tempo livre, coletivamente. A coleta de dados foi precedida da aplicação do Questionário de Dados Sociodemográficos.

\section{Procedimentos Éticos}

De acordo com a Resolução 466/12 do Conselho Nacional de Saúde referente à pesquisa com seres humanos, a presente pesquisa foi aprovada pelo Comitê de Ética em Pesquisa da Universidade e todos os participantes assinaram Termo de Consentimento Livre e Esclarecido (TCLE).

\section{Procedimentos de Análise de Dados}

Os dados foram analisados através do programa SPSS 20.0. Foi realizado o teste $t$ de Student para comparação dos grupos das instituições públicas e privadas. Foi utilizada a correlação de Pearson entre as dimensões do QVA-r, os fatores do IHS e o ICSA. O nível de significância adotado foi de $5 \%(\mathrm{p} \leq 0,05)$.

\section{APRESENTAÇÃO E DISCUSSÃO DE RESULTADOS}

A Tabela 1 mostra que os fatores do ICSA estão correlacionados com os fatores do QVA e do IHS. Há correlação positiva fraca entre o ICSA Habilidade em Sala de Aula e os fatores do IHS Enfrentamento com Risco, Autoafirmação na Expressão de Sentimento Positivo, Conversação e Desenvoltura Social e Autoafirmação na Expressão a Desconhecidos, bem como correlação positiva moderada com o total do IHS. Isto significa dizer que a habilidade para participar ativamente em sala de aula, discutindo e debatendo o conteúdo de uma disciplina, está correlacionada positivamente com as habilidades de defesa dos próprios direitos e de expressão de afeto positivo. Também implica ter desenvoltura e saber iniciar e manter uma conversação com pessoas conhecidas ou desconhecidas, transitando entre os diversos grupos. Nesse sentido, uma situação aparentemente tão simples como participar de uma aula necessita de relações interpessoais estabelecidas de forma que permitam ao estudante partilhar experiências, compartilhar dificuldades e assinalar situações em que percebe que seus direitos estão sendo de alguma forma violados, como apontam Teixeira, Dias, Wottrich e Oliveira (2008).

Foi verificada a existência de correlação negativa fraca entre o fator do ICSA Cordialidade no Relacionamento 


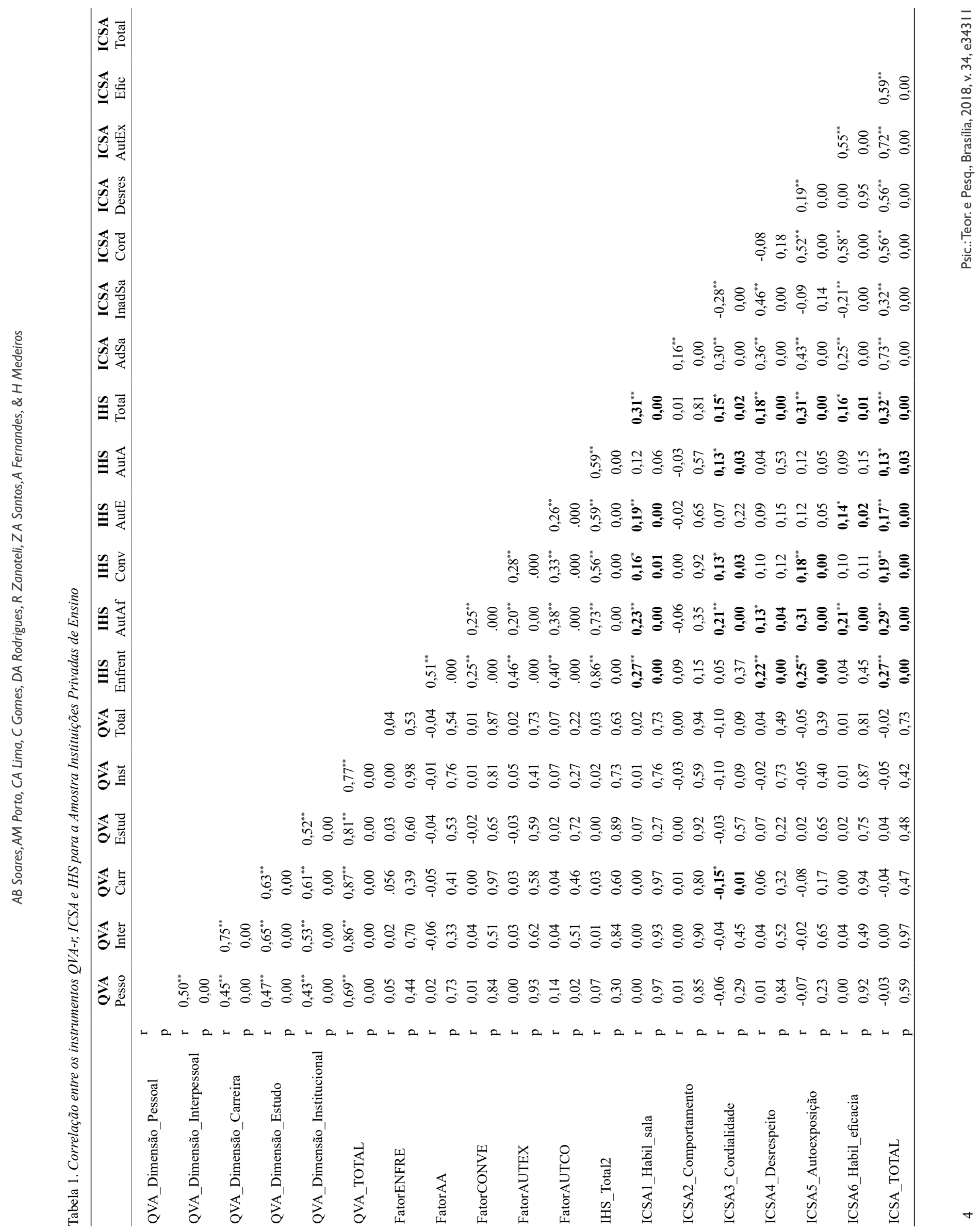


Interpessoal e o fator do QVA Carreira, bem como correlação positiva fraca com os fatores do IHS Autoafirmação na Expressão de Sentimento Positivo, Conversação e Desenvoltura Social, Autocontrole da Agressividade e o IHS Total. Tal correlação mostra que a habilidade para trabalhar em grupo, valorizando a cordialidade e a troca com as outras pessoas, em detrimento de uma visão mais individualista, está correlacionada com a percepção do estudante que se sente seguro em relação à sua opção vocacional e suas expectativas profissionais. Da mesma forma, essa habilidade para trabalhar em grupo implica que o estudante necessita se aproximar de pessoas, expressando opiniões e sentimentos positivos em relação às mesmas e, ao mesmo tempo, ter autocontrole emocional, principalmente no que diz respeito a sentimentos de raiva e tendências agressivas. Para Teixeira et al. (2008), os vínculos afetivos com os colegas são essenciais para a adaptação. Esses vínculos contribuem, junto com os próprios interesses profissionais, para o sucesso acadêmico (Noronha, Martins, Gurgel, \& Ambiel, 2009).

Há correlação positiva fraca entre o fator do ICSA Desrespeito a Professores e Colegas e os fatores do IHS Enfrentamento com Risco, Autoafirmação na Expressão de Sentimento Positivo e IHS Total. Isto significa que, quando o estudante tenta impor sua opinião a colegas e professores, assumindo uma postura competitiva e questionadora, parece estar mais concentrado na defesa de seus direitos e, ao mesmo tempo, na exposição de seus sentimentos, o que poderia caracterizar uma postura mais voltada para suas próprias necessidades. Nessas situações, o estudante pode contribuir para que o seu próprio processo de adaptação à universidade fique prejudicado, pois, como afirmam Noronha et al. (2009), os estudantes que investem nos relacionamentos com colegas e professores diferenciam-se por apresentarem uma maior adaptação, além de métodos de estudo mais eficientes e maior desenvolvimento vocacional.

Foi encontrada correlação positiva fraca entre o fator do ICSA Autoexposição e Assertividade e os fatores do IHS Enfrentamento com Risco e Conversação e Desenvoltura Social, bem como correlação positiva moderada com Autoafirmação na Expressão de Sentimento Positivo e IHS Total. Esse resultado aponta para o fato de que, quanto mais assertivo o estudante é, mais enfrenta as dificuldades que se interpõem em seu caminho. Por outro lado, deve também ter a habilidade de se conduzir nas diversas situações sociais e saber expressar, de forma socialmente competente, seus sentimentos positivos quando os mesmos surgem. Na realidade, para Santos e Almeida (2001), as tarefas desenvolvimentais com as quais o estudante se depara estão relacionadas com a aquisição de autonomia, desenvolvimento da identidade e gerenciamento das relações interpessoais, estando estas diretamente ligadas ao processo de aprendizagem.

Foi constatada também correlação positiva fraca entre o fator do ICSA Habilidade em Eficácia Acadêmica e os fatores do IHS Autoafirmação na Expressão de Sentimento
Positivo, Autoexposição a Desconhecidos e IHS Total, o que indica que, quanto mais o estudante consegue se adaptar a instituição e ao grupo, desenvolvendo competências acadêmicas que lhe permitam compreender o conteúdo ensinado, mais habilidade deve apresentar para expressar seus sentimentos e se expor, inclusive diante de pessoas desconhecidas. De acordo com Teixeira et al. (2008), os vínculos afetivos com os colegas, as relações com os professores, as atividades extraclasse e o desenvolvimento de estratégias para lidar com as frustrações e dificuldades são importantes na experiência de adaptação.

Foi encontrada correlação positiva fraca entre o fator do ICSA Total e os fatores do IHS Enfrentamento com Risco, Autoafirmação na Expressão de Sentimento Positivo, Conversação e Desenvoltura Social, Autoexposição a Desconhecidos e Autocontrole da Agressividade, assim como correlação positiva moderada com o fator IHS Total. Desses resultados, pode-se depreender que a habilidade para apresentar comportamentos sociais acadêmicos adequados de forma geral está correlacionada com a defesa de direitos interpessoais, mas também com a expressão de sentimentos positivos e com ter desenvoltura social em diversas situações. Finalmente, pode-se concluir que a estada na universidade constitui uma experiência impactante, em que o estudante necessita lidar com o ambiente universitário, com as características institucionais e, principalmente, com a necessidade de gerenciar situações que exigem respostas sociais habilidosas e adaptativas frente a situações novas e desafiadoras (Cruz \& Schultz, 2009).

Conforme pode ser observado na Tabela 2, na amostra das instituições públicas foram significativas as correlações entre os fatores Habilidade em Sala de Aula (ICSA) com a dimensão Pessoal do QVAr. A correlação negativa moderada significa que o formato requerido para a adaptação às exigências em sala de aula pode levar o aluno a variações de humor, à angústia, à ansiedade e ao cansaço. $\mathrm{O}$ estudo mostrou que os estudantes das universidades públicas que apresentaram comportamentos adequados em sala de aula tiveram dificuldades pessoais de autonomia, bem-estar físico, bem-estar psicológico e percepção pessoal de competências cognitivas. Miyasaki (1997) corrobora esses achados ao afirmar que os alunos que são perfeccionistas se cobram muito, não aceitam fracassar e possuem alto grau de exigência. Caso falhem, apresentam sentimentos de descontrole, desespero, ansiedade, medo e raiva. Portanto, o aluno que é muito exigente consigo mesmo, embora apresente habilidades em sala de aula, pode ter desgaste pessoal para administrar as atividades acadêmicas. As habilidades em sala de aula exigem do aluno comportamentos adequados que requerem dedicação, tempo disponível, responsabilidade e comprometimento, o que pode trazer prejuízos para a qualidade de vida pessoal.

O fator Comportamento Indisciplinado em Sala de Aula (ICSA) apresentou uma correlação negativa moderada com a dimensão Estudo do QVAr, ou seja, quanto mais o 
comportamento do aluno é inadequado em sala de aula, mais dificuldades apresenta na administração do tempo, no planejamento diário de suas atividades, na preparação para as provas, apresentando, enfim, piores métodos de estudo. Esses alunos mostraram dificuldades em atividades curriculares, o que pode evidenciar que a adaptação acadêmica pode depender de condições ambientais e sociais, entre outras. Segundo Luz, Castro, Couto, Santos e Pereira (2009), ao ingressar na universidade, o estudante está exposto a situações estressoras que terá que administrar. Porém, o ambiente acadêmico pode contribuir para solucionar tais dificuldades, sendo considerado um ambiente facilitador ao desenvolvimento e propício para o ajustamento acadêmico, pessoal, social e afetivo, constituindo-se em um suporte durante a formação acadêmica (Ferreira, Almeida, \& Soares, 2001). Para que o aluno se dedique aos estudos, é fundamental que o comportamento em sala de aula seja adequado. Ser indisciplinado implica desatenção, desrespeito, intolerância, incapacidade de ser empático, baixa tolerância à frustração e pouco aprendizado (Almeida et al., 2007).

$\mathrm{O}$ fator pertinente à Cordialidade no Relacionamento Interpessoal (ICSA) apresentou uma correlação fraca com as dimensões Carreira do QVAr e Estudo, transparecendo que os comportamentos de cordialidade no ambiente acadêmico se associam a expectativas vocacionais que envolvem o próprio curso, a carreira e a futura profissão. O fator Cordialidade (ICSA) apresentou uma correlação positiva fraca com o QVAr Total, evidenciando também uma associação entre esses comportamentos e as vivências acadêmicas em geral. $\mathrm{E}$, ainda, o fator Cordialidade apresentou uma correlação negativa moderada com o Enfrentamento e a Autoafirmação com Risco do IHS, mostrando que a paciência com os colegas, a valorização do grupo e o bom senso em geral dificultam a expressão do comportamento assertivo em relação a si mesmo, em situações de exposição perante ao outro e ao grupo. O estudo de Teixeira et al. (2007) mostrou que a dimensão Interpessoal, inerente às habilidades de fazer amizades, expressar sentimentos, possuir bom nível de tolerância intercultural e capacidade de cooperar com os colegas, correlaciona-se com a dimensão Carreira do QVAr. Os estudantes que apresentam um bom relacionamento interpessoal investem mais no curso que escolheram, organizam projetos e ambicionam sucesso em relação a sua profissão.

O fator Desrespeito a Professores e Colegas do ICSA apresenta uma correlação positiva moderada com o fator Enfrentamento e Autoafirmação com Risco do IHS, o que implica que, quanto mais o aluno for desrespeitoso, expondo colegas e professores, questionando o professor e interrompendo colegas e professores, mais dificuldade apresenta para mostrar comportamentos assertivos que envolvam a compreensão de direitos e deveres do estudante. O estudo de Silva e Nascimento (2012) procurou compreender a motivação e os processos de integração dos estudantes à Universidade e os resultados apontam na mesma direção deste estudo. Os autores identificaram que as relações interpessoais dos estudantes com os professores e os colegas podem contribuir para adaptação ao Ensino Superior. Comportamentos do estudante como o desrespeito com o professor e a outros alunos estão correlacionados com o comportamento de afirmação e defesa de direitos e de autoestima, com risco potencial de reação indesejável.

O fator Autoexposição e Assertividade (ICSA) apresentou correlação negativa fraca com o QVA-r - dimensão Pessoal, evidenciando que demonstrar comportamentos assertivos de autoexposição pode ter consequências indesejáveis e pode conduzir ao cansaço, à ansiedade, a variações de humor e ao pessimismo. Entretanto, esse fator também encontrou uma correlação positiva fraca com a dimensão Estudo, o que sugere que o aluno assertivo apresenta boas rotinas de estudos, gestão de tempo, utilização da biblioteca, utilização do laboratório de informática e de outros recursos de aprendizagem. Teixeira et al. (2007) detectaram correlações entre a dimensão Interpessoal e Carreira do QVA-r e identificaram que os estudantes percebem que as relações interpessoais acadêmicas promovem uma possiblidade de estabelecerem um futuro profissional com sucesso.

Ao analisar o fator Habilidade em Eficácia Acadêmica (ICSA) e a dimensão Estudo (QVAr), encontra-se uma correlação positiva moderada. Entre o fator Habilidade em Eficácia Acadêmica (ICSA) e o QVAr total, encontrase uma correlação positiva fraca. Expressa-se, assim, que o universitário que apresenta um bom desempenho acadêmico apresenta bons métodos de estudo e bem-estar psicológico e físico, autoconfiança e autonomia. Percebe-se que o ICSA Total apresentou correlação negativa com o QVAr - dimensão Pessoal, indicando que o comportamento social acadêmico adequado pode levar a dificuldades de concentração, cansaço e ansiedade. Segundo Gerk e Cunha (2006), o sucesso acadêmico se fundamenta em algumas características pessoais, grau de adaptabilidade, conhecimentos, habilidades e atitudes, confirmando que o desempenho do universitário está pautado em comportamentos adequados de estudo e características psicológicas. O estudo de Cunha e Carrilho (2005) indica que os alunos com as melhores vivências acadêmicas na dimensão Pessoal e de Realização Acadêmica apresentam melhor rendimento escolar, ou seja, o crescimento do estudante em relação a si próprio e aos objetivos propostos pela instituição universitária pode ser considerado como seu sucesso acadêmico, vindo a confirmar os resultados deste estudo.

De maneira geral, as dimensões do ICSA apresentaram mais correlações com o IHS na amostra das instituições particulares e mais correlações com o QVAr na amostra das instituições públicas. Considerando os comportamentos sociais acadêmicos dos alunos das universidades particulares e públicas em decorrência de mudanças socioeconômicas, o estudo de Soares, Mello e Baldez (2011) identificou uma 


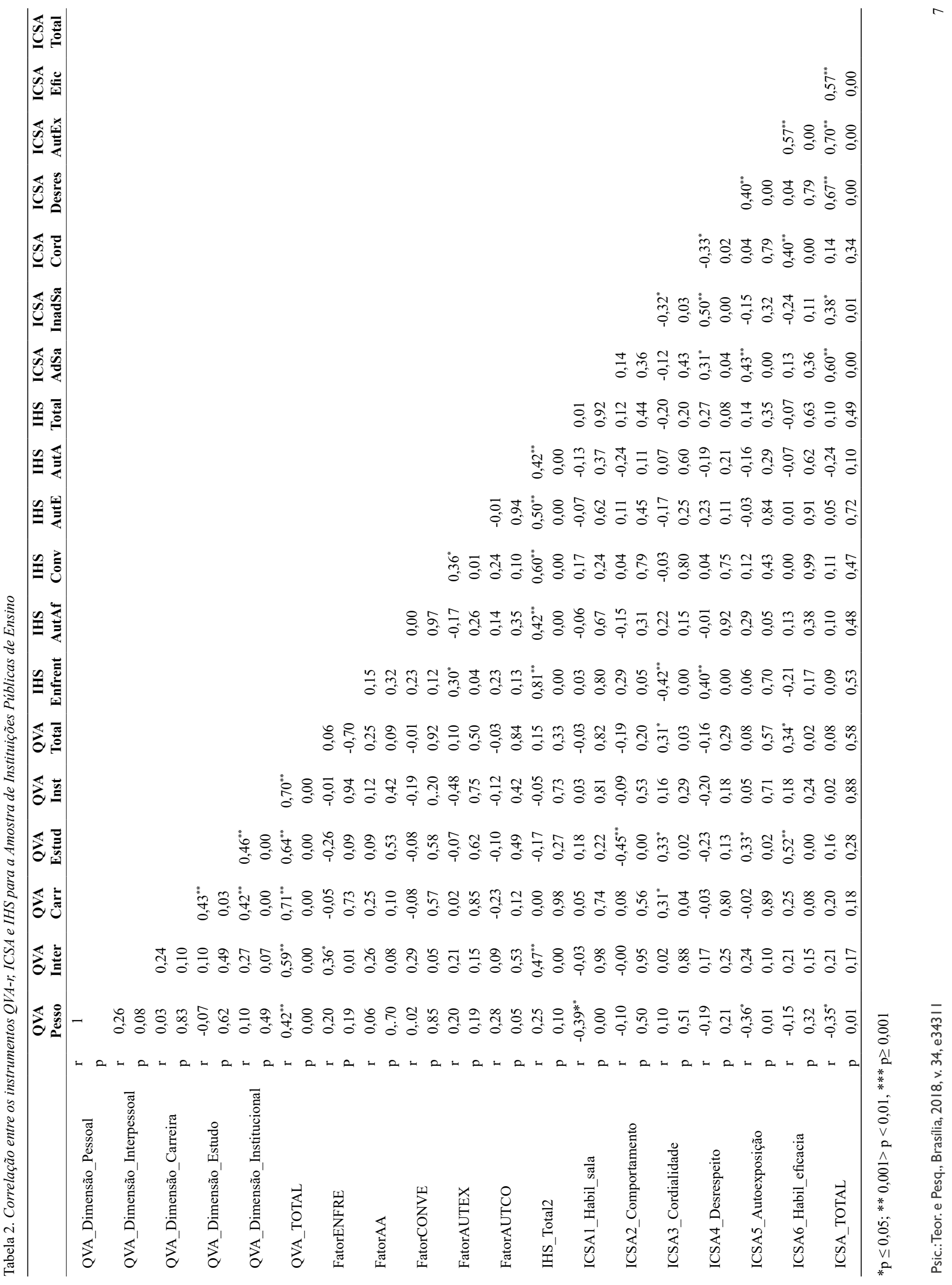


maior autonomia dos alunos que estudam em faculdades particulares. Esses achados confirmam os resultados deste estudo. Em pesquisa de Soares, Gomes e Seabra (2014) com universitários do primeiro ano, verificou-se que os estudantes da rede particular apresentam escores de vivências acadêmicas superiores aos da rede pública, além da diferença entre as médias nos fatores do IHS e no escore total serem significativamente superiores para os estudantes universitários de instituições particulares, transparecendo que os universitários de rede privada apresentam vivências acadêmicas e repertório de HS mais elaborado, o que poderia facilitar a adaptação acadêmica.

Como pode ser observado na Tabela 3, a diferença entre as médias obtidas no QVA-r pelos indivíduos de acordo com a Instituição foi significativa nas dimensões Interpessoal, Carreira, Institucional, QVA-r Total e no fator Autocontrole da Agressividade do IHS em que os indivíduos pertencentes a instituições públicas obtiveram em média, escores superiores àqueles pertencentes às privadas.

No que diz respeito à dimensão Interpessoal, pode-se dizer que os estudantes das instituições públicas apresentam mais facilidade no relacionamento interpessoal com amigos, colegas e professores. Em um estudo correlacional com estudantes universitários usando o QVA-r, Teixeira et al. (2007) verificaram que foram as dimensões Carreira e Interpessoal aquelas que apresentaram médias mais altas, revelando satisfação com o curso e com as relações pessoais estabelecidas no âmbito da universidade. No mesmo estudo, foi observado que a dimensão Carreira obteve uma correlação forte com a dimensão Institucional, o que, segundo os autores, sugere que a avaliação que o sujeito faz da satisfação com sua escolha e curso relaciona-se diretamente com a avaliação global que o indivíduo faz da instituição na qual estuda. Adicionado a isso, tal estudo também apontou que a correlação entre as dimensões Interpessoal e Carreira indicaram a importância das amizades estabelecidas no meio universitário para o sentimento de satisfação com o curso e a profissão. Conforme Granado (2004), a dimensão Carreira mede o quanto os estudantes acreditam que, no futuro, poderão ter a oportunidade de concretizar expectativas e valores vocacionais, bem como obter realização profissional. Nesse sentido, o estudo de Sarriera, Paradiso, Schütz e Howes (2012) mostrou que essa dimensão apresentou a média mais alta nas três instituições investigadas, entre duas instituições públicas e uma privada. No entanto, estudantes de universidades privadas apresentaram maiores escores de vivências acadêmicas avaliadas pela dimensão Carreira. Tal achado contraria o resultado da presente pesquisa, segundo a qual a diferença das médias entre as instituições mostrou-se a favor da universidade pública. Desse modo, alunos da universidade pública acreditam estar vocacionados e identificados com a carreira que escolheram e também acreditam na sua realização profissional. Tal estudo pode indicar que os alunos das universidades públicas sentem-se mais identificados com a carreira que escolheram e têm maiores expectativas de realização profissional.

Embora, como foi apresentado também por Sarriera et al. (2012), haja uma lacuna observada nas investigações quanto à variável instituição, uma vez que poucos estudos são realizados com estudantes de diferentes universidades (Granado et al., 2005; Soares et al., 2009), as instituições públicas apresentaram médias significativamente maiores do que as privadas na dimensão Institucional. Tal resultado corrobora o presente estudo, em que é apresentada uma diferença de média (mais alta para instituições públicas) e é indicado que alunos dessas instituições estão mais alinhados com a universidade que frequentam, conhecem e utilizam os recursos institucionais.

No que diz respeito às HS, os estudantes do ensino público apresentaram maior autocontrole da agressividade, conseguindo lidar melhor com as críticas, em geral, e com a dos pais, em especial. Estudo realizado por Tavares, Couto e Silva (2012) mostrou que estudantes universitários de psicologia também apresentaram uma diferença significativa no fator Autocontrole da Agressividade, o que significa dizer que tais estudantes apresentam maior controle da raiva e da agressividade quando são interpelados por críticas, podendo apresentar, assim, maior desenvoltura social em situações sociais variadas. 
Tabela 3. Análise das diferenças dos escores fatoriais e totais dos instrumentos QVA-r, ICSA e IHS para instituições públicas e privadas

\begin{tabular}{|c|c|c|c|c|c|}
\hline Variável & Instituição & Média & Desvio Padrão & $t$ & $\mathbf{p}$ \\
\hline \multirow{2}{*}{ QVA Pessoal } & Privada & 3,41 & 0,69 & $-0,34$ & 0,729 \\
\hline & Pública & 3,45 & 0,62 & & \\
\hline \multirow{2}{*}{ QVA_Interpessoal } & Privada & 3,54 & 0,77 & $-2,85$ & $0,006 * *$ \\
\hline & Pública & 3,83 & 0,58 & & \\
\hline \multirow{2}{*}{ QVA Carreira } & Privada & 3,61 & 0,89 & $-2,72$ & $0,008 * *$ \\
\hline & Pública & 3,92 & 0,67 & & \\
\hline \multirow{2}{*}{ QVA Estudo } & Privada & 3,31 & 0,76 & $-0,89$ & 0,373 \\
\hline & Pública & 3,42 & 0,68 & & \\
\hline \multirow{2}{*}{ QVA Institucional } & Privada & 3,35 & 0,79 & $-3,94$ & $0,000 * * *$ \\
\hline & Pública & 3,73 & 0,52 & & \\
\hline \multirow{2}{*}{ QVA TOTAL } & Privada & 17,25 & 3,16 & $-3,20$ & $0,002 * *$ \\
\hline & Pública & 18,37 & 1,90 & & \\
\hline \multirow{2}{*}{ IHS Enfrentamento } & Privada & 10,32 & 2,98 & 0,91 & 0,362 \\
\hline & Pública & 9,96 & 2,23 & & \\
\hline \multirow{2}{*}{ IHS Autoafirmação } & Privada & 8,59 & 1,92 & $-1,87$ & 0,062 \\
\hline & Pública & 9,16 & 1,46 & & \\
\hline \multirow{2}{*}{ IHS Conversação } & Privada & 6,36 & 1,46 & $-1,12$ & 0,264 \\
\hline & Pública & 6,62 & 1,27 & & \\
\hline \multirow{2}{*}{ IHS Autoexposiçao } & Privada & 3,21 & 1,09 & $-0,50$ & 0,615 \\
\hline & Pública & 3,30 & 1,11 & & \\
\hline \multirow{2}{*}{ IHS Autocontrole } & Privada & 2,84 & 0,82 & $-4,92$ & $0,000 * * *$ \\
\hline & Pública & 3,34 & 0,56 & & \\
\hline \multirow{2}{*}{ IHSTotal } & Privada & 31,43 & 5,99 & $-1,24$ & 0,216 \\
\hline & Pública & 32,36 & 4,03 & & \\
\hline \multirow{2}{*}{ ICSA ComportamentoAdequa } & Privada & 2,93 & 0,73 & $-0,29$ & 0,765 \\
\hline & Pública & 2,95 & 0,53 & & \\
\hline \multirow{2}{*}{ ICSA ComportInd } & Privada & 2,48 & 0,70 & 1,61 & 0,107 \\
\hline & Pública & 2,30 & 0,66 & & \\
\hline \multirow{2}{*}{ ICSA Cordialidade } & Privada & 3,92 & 0,64 & $-1,89$ & 0,059 \\
\hline & Pública & 4,11 & 0,49 & & \\
\hline \multirow{2}{*}{ ICSA Desrespeito } & Privada & 2,45 & 0,65 & 0,03 & 0,971 \\
\hline & Pública & 2,45 & 0,62 & & \\
\hline \multirow{2}{*}{ ICSA Autoexposição } & Privada & 3,41 & 0,62 & $-1,27$ & 0,202 \\
\hline & Pública & 3,54 & 0,50 & & \\
\hline \multirow{2}{*}{ ICS Habilidade de Eficácia } & Privada & 3,85 & 0,63 & $-0,71$ & 0,473 \\
\hline & Pública & 3,93 & 0,57 & & \\
\hline \multirow{2}{*}{ ICSA TOTAL } & Privada & 19,07 & 2,33 & $-0,63$ & 0,527 \\
\hline & Pública & 19,31 & 1,75 & & \\
\hline
\end{tabular}

$* \mathrm{p} \leq 0,05 ; * * 0,001>\mathrm{p}<0,01 * * * \mathrm{p} \geq 0,001$ 


\section{CONSIDERAÇÕES FINAIS}

O presente estudo teve como objetivo identificar as relações entre HS, vivências acadêmicas e comportamentos sociais acadêmicos em estudantes universitários do estado do Rio de Janeiro. O estudo identificou que os alunos que apresentam comportamentos sociais acadêmicos desadaptativos também apresentam déficits em HS, prejudicando diretamente suas vivências na instituição e dificultando sua adaptação acadêmica. $O$ estudo mostrou que habilidades socialmente aceitáveis para uma vida acadêmica estão associadas não só a varáveis pessoais, mas também a quesitos curriculares e institucionais.

$\mathrm{O}$ estudante pode vivenciar mais tranquilamente a universidade quando troca experiências e estabelece relações interpessoais com os colegas e professores. A instituição, por sua vez, possui também uma grande parcela de responsabilidade em integrar o aluno e propiciar o seu desenvolvimento para que, ao término do curso, tenha adquirido as habilidades e competências necessárias para exercer sua profissão.

$\mathrm{O}$ estudo corroborou pesquisas recentes que enfatizam a importância de competências em HS e êxito acadêmico. Entretanto, comportamentos sociais acadêmicos adequados podem também trazer prejuízos para o aluno que se priva de uma vida social para melhorar o seu desempenho acadêmico e, com isso, podem provocar alterações de humor, como estresse, angústia e ansiedade. Alguns estudantes não conseguem administrar o tempo de estudos adequadamente, acarretando prejuízos na vida acadêmica e social.

$\mathrm{O}$ estudo apresentou como limitação um número pouco expressivo de estudantes de instituições públicas. Sugere-se que estudos posteriores levem em consideração essas diferentes realidades, assim como os períodos que os estudantes estão cursando, uma vez que, a cada semestre, mais experiências são realizadas.

\section{REFERÊNCIAS}

Almeida, L. S., \& Soares, A. P. (2003). Os estudantes universitários: Sucesso escolar e desenvolvimento psicossocial. In E. Mercuri \& S. A. J. Polydoro (Eds.), Estudante universitário: Características e experiências de formação (pp. 15-40). Taubaté: Cabral Editora e Livraria Universitária.

Almeida, L. S., Soares, A. P., \& Ferreira, J. A. (2000). Transição e adaptação à universidade: Apresentação de um Questionário de Vivências Académicas (QVA). Psicologia, 14(2), 189208.

Almeida, L. S., Soares, A. P., Guisande, A. A., \& Paisana, J. (2007). Rendimento acadêmico no ensino superior: Estudo com alunos do $1^{\circ}$ ano. Revista Galego-Portuguesa de Psicoloxía e Ecaducación, 14, 207-220.

Almeida, L.S., Soares, A.P.C., \& Ferreira, J. A. (2002). Questionário de Vivências Acadêmicas: Avaliação do ajustamento dos estudantes universitários. Avaliação Psicológica, 1(2), 81-93.

Alves, T. W., \& Alves, V.V. (2009). Fatores determinantes da evasão universitária: Uma análise a partir dos alunos da UNISINOS. Revista Contemporânea de Economia e Gestão, 10(2), 115-129.

Bandeira, M., \& Quaglia, M. A. C. (2006). Comportamento assertivo: Relações com ansiedade, lócus de controle e autoestima. (2006). In M. Bandeira, A. Del Prette, \& Z. A. P. Del Prette (Eds.), Estudo sobre habilidades sociais e relacionamento interpessoal (pp. 161-179). São Paulo: Casa do Psicólogo.

Bariani, I. C. D., \& Pavani, R. (2008). Sala de aula na universidade: Espaço de relações interpessoais e participação acadêmica. Estudos de Psicologia (Campinas), 25(1), 67-75.

Costa, L., \& Oliveira, M. (2010). Vivências e satisfação académica em alunos do ensino superior. Cadernos de Pedagogia no Ensino Superior, 13, 13-32.

Cruz, R. M., \& Schultz, V. (2009). Avaliação de competências profissionais e formação de psicólogos. Arquivos Brasileiros de Psicologia, 61(3), 117-127.

Cunha, S. M., \& Carrilho, D. M. (2005). O processo de adaptação ao ensino superior e o rendimento acadêmico. Psicologia Escolar e Educacional, 9(2), 215-224.

Del Prette, Z. A. P., \& Del Prette, A. (2001). Inventário de Habilidades Sociais (IHS-Del Prette): Manual de aplicação, apuração e interpretação. São Paulo: Casa do Psicólogo.
Del Prette, Z. A. P., \& Del Prette, A. (2004). Desenvolvimento interpessoal: Uma questão pendente no universitário. In E. Mercuri, \& S. A. J. Polydoro (Eds.), Estudante universitário: Características e experiências de formação (pp. 105-128). Taubaté: Cabral Editora e Livraria Universitária.

Feitosa, F. B., Matos, M. G., Del Prette, Z. A. P., \& Del Prette, A. (2009). Desempenho acadêmico e interpessoal em adolescentes portugueses. Psicologia em Estudo, 14(2), 259-266.

Ferreira, J. A., Almeida, L. S., \& Soares, A. P. (2001). Adaptação acadêmica em estudantes do $1^{\circ}$ ano: Diferenças de gênero, situação de estudantes e cursos. Revista Psico-USF, 6, 1-10.

Gerk, E., \& Cunha, S. (2006). Habilidades sociais na adaptação de alunos estudantes ao ensino superior. In M. Bandeira, A. Del Prette, \& Z. A. P. Del Prette (Eds.), Estudo sobre habilidades sociais e relacionamento interpessoal (pp. 181-198). São Paulo: Casa do Psicólogo.

Granado, J. I. F. (2004). Vivência acadêmica de universitários brasileiros: Estudo de validade e precisão do QVA-r (Dissertação de mestrado não publicada). Universidade São Francisco, Itatiba, SP, Brasil.

Granado, J. I. F., Santos, A. A. A., Almeida, L. S., Soares, A. P., \& Guisande, M. A. (2005). Integração acadêmica de estudantes universitários: Contributos para a adaptação e validação do QVA-r no Brasil. Psicologia e Educação, 4(2), 31- 41.

Gresham, F. M. (2009). Evolution of the treatment integrity concept: Current status and future directions. School Psychology Review, 38(4), 533-540.

Kanan, L. A., \& Zanelli, J. C. (2011). Envolvimento de docentesgestores com o trabalho no contexto universitário. Psicologia \& Sociedade, 23(1), 56-65.

Luz, A., Castro, A., Couto, D., Santos, L., \& Pereira, A. (2009). Stress e percepção do rendimento académico no aluno do ensino superior. In Actas do Décimo Congresso Internacional Galego-Português de Psicopedagogia (pp. 4663-4669).

Mercuri, E., \& Polydoro, S. A. J. (2003) O compromisso com o curso no processo de permanência/evasão no ensino superior: Algumas contribuições. In E. Mercuri \& S. A. J. Polydoro (Eds.), Estudante universitário: Características e experiências 
de formação (pp. 219-236). Taubaté: Cabral Editora e Livraria Universitária.

Miller, T. E. (2005). Introdution. In B. E. Miller, B. E. Bender, \& J. H. Schuh (Eds.), Promoting reasonable expections: Aligining student and instituitional views of the college experience (pp. 1-9). San Francisco, CA: Jossey-Bass.

Miyazaki, M. C. O. S. (1997). Psicologia na formação médica: Subsídios para prevenção e trabalho clínico com universitários (Tese de doutorado). Universidade de São Paulo, São Paulo, SP, Brasil.

Noronha, A. P. P., Martins, D. F., Gurgel, M. G. A., \& Ambiel, R. A.M. (2009). Estudo correlacional entre interesses profissionais e vivências acadêmicas no ensino superior. Psicologia Escolar e Educacional, 13, 143-154.

Nunes, G. T. (2005). Abordagem do Marketing de Relacionamento no Ensino Superior: Um estudo exploratório (Dissertação de mestrado). Universidade Federal de Santa Catarina, Florianópolis, SC, Brasil.

Parker, J. D. A., Summerfeldt, L. J., Hogan, M. J., \& Majeski, S. (2004). Emotional intelligence and academic success: Examining the transition from high school to university. Personality and Individual Differences, 36, 163-172.

Santos, A. A. A., Noronha, A. P. P., Amaro, C. B., \& Villar, J. (2005). Questionário de Vivência Acadêmica: Estudo de consistência interna do instrumento no contexto brasileiro. In M. C. R. A. Joly, A. A. A dos Santos, \& F. F. Sisto (Eds.), Questões do cotidiano universitário (pp. 159-177). São Paulo: Casa do Psicólogo.

Santos, L., \& Almeida, L. S. (2001) Vivências acadêmicas e rendimento escolar: Estudo com alunos universitários do $1^{\circ}$ ano. Análise Psicológica, 19, 205-217.

Sarriera, J. C., Paradiso, A. C., Schütz, F. F., \& Howes, P. (2012). Estudo comparativo da integração ao contexto universitário entre estudantes de diferentes instituições. Revista Brasileira de Orientação Profissional, 13(2), 163-172.

Silva, R. S., \& Nascimento, I. (2012). Estudantes maiores de 23 anos em Psicologia: Motivações e processo de integração. Revista Brasileira de Orientação Profissional, 13, 1, 37-47.

Silva, S. S., \& Fleith, D. S. (2005). Desempenho escolar e autoconceito de alunos atendidos em serviços psicopedagógicos. Psicologia Escolar e Educacional, 9(2), 235-245.
Soares, A. B., Gomes, G., \& Seabra, A. M. R. (2014). Inteligência, autoeficácia e habilidades sociais em estudantes universitários, Revista Brasileira de Orientação Profissional, 15(1), 85-94.

Soares, A., Mello, T., \& Baldez, M. (2011). Vivências acadêmicas em estudantes universitários do estado do Rio de Janeiro. Interação em Psicologia, 15(1), 59-69.

Soares, A. B., Mourão, L., \& Mello, T. V. S. (2011). Estudo para a construção de um instrumento de comportamentos acadêmicosociais para estudantes universitários. Estudos e Pesquisas em Psicologia, 11(2), 488-506.

Soares, A. B., Poubel, L. N., \& Mello, T. V. S. (2009). Habilidades sociais e adaptação acadêmica: Um estudo comparativo em instituições de ensino público e privado. Aletheia, 29, 27-42.

Tavares, W. M., Couto, G., \& Silva, R. L. F. (2012). Perfil das relações interpessoais e habilidades sociais de estudantes de Psicologia. Estudos Interdisciplinares em Psicologia, 3(1), 75-92.

Teixeira, M. A. P., Castro, G. D., \& Piccolo, L. R. (2007). Adaptação à universidade em estudantes universitários: Um estudo correlacional. Interação em Psicologia, 11(2), 211-220.

Teixeira, M. A. P., Dias, A. C. G., Wottrich, S. H., \& Oliveira, A. M. (2008). Adaptação à universidade em jovens calouros. Psicologia Escolar e Educacional, 12(1), 185-202.

Tinto, V. (2012). Enhancing student success: Taking the classroom success seriously. The International Journal of the First Year in Higher Education, 3(1), 1-8. Retirado de https://fyhejournal. com/article/view/119/120.

Vendramini, C. M. M., Santos, A. A. A., Polydoro, S. A. J., Sbardelini, E. T. B., Serpa, M. N. F., \& Natário, E. G. (2004). Construção e validação de uma escala sobre avaliação da vida acadêmica. Estudos de Psicologia, 9(2), 259-268.

Villar, J. D., \& Santos, A. A. A. (2001). Questionário de vivência universitária: Adaptação para estudantes brasileiros (Manuscrito não publicado). Universidade São Francisco, Itatiba, SP, Brasil. 